OPEN ACCESS

Edited by:

Tiziana Rancati,

Istituto Nazionale dei Tumori (IRCCS),

Italy

Reviewed by: Gilles Defraene,

KU Leuven, Belgium

Irina Vergalasova,

Rutgers Cancer Institute of New

Jersey, United States

${ }^{*}$ Correspondence:

Jianbin $\mathrm{Li}$

lijianbin@msn.com

Specialty section:

This article was submitted to

Radiation Oncology,

a section of the journal

Frontiers in Oncology

Received: 18 August 2020 Accepted: 04 November 2020 Published: 17 December 2020

Citation:

Du F, Tang N, Cui Y, Wang W, Zhang Y, Li Z and Li J (2020) A Novel Nomogram Model Based on Cone-

Beam CT Radiomics Analysis Technology for Predicting Radiation Pneumonitis in Esophageal Cancer Patients Undergoing Radiotherapy.

Front. Oncol. 10:596013.

doi: 10.3389/fonc.2020.596013

\section{A Novel Nomogram Model Based on Cone-Beam CT Radiomics Analysis Technology for Predicting Radiation Pneumonitis in Esophageal Cancer Patients Undergoing Radiotherapy}

\author{
Feng $\mathrm{Du}^{1,2}$, Ning Tang ${ }^{1}$, Yuzhong $C u i^{3}$, Wei Wang ${ }^{3}$, Yingjie Zhang $^{3}$, Zhenxiang $L i^{3}$ \\ and Jianbin $\mathrm{Li}^{3 *}$
}

${ }^{1}$ Department of Radiation Oncology, School of Clinical Medicine, Cheeloo College of Medicine, Shandong University, Jinan, China, ${ }^{2}$ Department of Radiation Oncology, Zibo Municipal Hospital, Zibo, China, ${ }^{3}$ Department of Radiation Oncology, Shandong Cancer Hospital and Institute, Shandong First Medical University and Shandong Academy of Medical Sciences, Jinan, China

Purpose: We quantitatively analyzed the characteristics of cone-beam computed tomography $(\mathrm{CBCT})$ radiomics in different periods during radiotherapy $(\mathrm{RT})$ and then built a novel nomogram model integrating clinical features and dosimetric parameters for predicting radiation pneumonitis (RP) in patients with esophageal squamous cell carcinoma (ESCC).

Methods: At our institute, a retrospective study was conducted on 96 ESCC patients for whom we had complete clinical feature and dosimetric parameter data. CBCT images of each patient in three different periods of RT were obtained, the images were segmented using both lungs as the region of interest (ROI), and 851 image features were extracted. The least absolute shrinkage selection operator (LASSO) was applied to identify candidate radiomics features, and logistic regression analyses were applied to construct the radscore. The optimal period for the rad-score, clinical features, and dosimetric parameters were selected to construct the nomogram model and then the receiver operating characteristic $(\mathrm{ROC})$ curve was used to evaluate the prediction capacity of the model. Calibration curves and decision curves were used to demonstrate the discriminatory and clinical benefit ratios, respectively.

Results: The relative volume of total lung treated with $\geq 5$ Gy (V5), mean lung dose (MLD), and tumor stage were independent predictors of RP and were finally incorporated into the nomogram. When the three time periods were modeled, the first period was better than the others. In the primary cohort, the area under the ROC curve (AUC) was 0.700 (95\% confidence interval (Cl) 0.568-0.832), and in the independent validation cohort, the AUC was 0.765 (95\% Cl 0.588-0.941). In the nomogram model that integrates clinical features and dosimetric parameters, the AUC in the primary cohort was $0.836(95 \% \mathrm{Cl} 0.700$ 0.918), and the AUC in the validation cohort was 0.905 (95\% Cl 0.799-1.000). The 
nomogram model exhibits excellent performance. Calibration curves indicate a favorable consistency between the nomogram prediction and the actual outcomes. The decision curve exhibits satisfactory clinical utility.

Conclusion: The radiomics model based on early lung CBCT is a potentially valuable tool for predicting RP. V5, MLD, and tumor stage have certain predictive effects for RP. The developed nomogram model has a better prediction ability than any of the other predictors and can be used as a quantitative model to predict RP.

Keywords: esophageal cancer, cone beam computed tomography, radiation pneumonitis, prediction model, radiomics

\section{INTRODUCTION}

Among malignant tumors, the incidence rate of esophageal cancer (EC) is the seventh highest, and the mortality rate is sixth worldwide (1). Radiotherapy (RT) is still one of the main treatments for locally advanced EC $(2,3)$. However, radiation pneumonitis (RP) is one of the major toxicities of thoracic radiation therapy. If RP occurs, it seriously affects the patient's quality of life and survival prognosis (4). Therefore, it is imperative for EC patients undergoing RT to identify this toxicity at the earliest possible time. More importantly, the accurate prediction of RP is essential to facilitate individualized radiation dosing that leads to maximized therapeutic gain. At present, the risk assessment of RP is mainly predicted by using lung dosimetric parameters $(5,6)$, such as the relative volume of total lung irradiated above a specified threshold dose $\left(\mathrm{V}_{\mathrm{X}}\right)$ or mean lung dose (MLD): Although several metrics have appeared promising, the results vary across institutions, so these metrics do not seem to be perfect at predicting $\operatorname{RP}(7,8)$. In addition to dosimetric parameters, some clinical features (tumor stage, smoking history, preexisting lung diseases, concurrent chemotherapy, and radiation dose) are also considered to be related to RP occurrence. However, the consensus on the comparative importance of these related predictors remains unavailable at present. Consequently, in order to individually and precisely discern $\mathrm{RP}$, an accurate predictive model incorporating multiple types of factors with superior clinical utility is urgently needed.

Computed tomography (CT) images play an essential role in the diagnosis and treatment of RP. As early as the end of the 20th century, RP could be identified by CT. However, RP cannot be predicted by superficial CT manifestations. Therefore, the focus of later research is on the accurate prediction of RP (9). In recent years, with the rapid development of radiomics analysis technology, increasing attention has been paid to the research of RT effect and side effect predictions based on radiomics features (10-13). Among them, one study found that there is a dose-dependent relationship between the changes in some radiomics features and $\mathrm{RP} \geq 2$ grade determined by extracting local lung CT images after RT (12). Another study successfully established a differential model of high- and low-risk RP by analyzing the region of interest (ROI) of the whole lung tissue before RT (13). In short, radiomics features can capture the capability of lung texture features, which help describe the potential RP risk $(14,15)$.

At present, cone-beam computed tomography (CBCT) has become a routine online method of image-guided radiotherapy (IGRT) for EC. If we can perform quantitative analysis on CBCT radiomics features in a certain period of RT and then combine these radiomics features with clinical features and dosimetric parameters to predict RP in EC, it will help guide clinical treatment strategies in a timely manner.

Therefore, the initial aim of this study was to investigate whether the early changes in CBCT radiomics features could be used as potential markers for predicting RP. In the present study, a comprehensive nomogram, which is a conveniently applicable predictive model integrating CBCT radiomics features, clinical features, and dosimetric parameters, was built for the individualized risk assessment and precise prediction of RP.

\section{MATERIALS AND METHODS}

\section{Patients}

The entire cohort of this retrospective study was obtained from the records of our institutional picture archiving and communication system (PACS) from January 2017 to June 2019, which was used to identify esophageal squamous cell carcinoma (ESCC) patients receiving RT treatment. The inclusion criteria were as follows: (1) Karnofsky performance score (KPS) $\geq 70$, (2) no previous history of thoracic RT, (3) intensity-modulated radiotherapy (IMRT) and received $\geq 50$ Gy $\mathrm{RT}$, and (4) CBCT scan performed at least once a week during $\mathrm{RT}$ with the scanning range of the CBCT imaging including at least two thirds of the lungs. The exclusion criteria were as follows: (1) low image quality, (2) general pulmonary infection unrelated to RT, and (3) treatment break of more than 7 days during RT. A total of 96 consecutive patients with thoracic middle segment ESCC were identified and divided into two cohorts at a 7:3 proportion using computer-generated random numbers. Sixty-seven patients were allocated to the primary cohort, and 29 patients were allocated to the verification cohort. Our institutional research ethics board approved this retrospective study (SDTHEC201703014). It waived the need to obtain informed consent from the patients due to the retrospective nature of the investigation (retrospective single-institution cohort study). 


\section{Clinical Data and RT Parameters}

The clinical data were all acquired from the institute's medical records. Specifically, clinicopathological parameters included age, sex, KPS, smoking status, diabetes history, chronic obstructive lung disease (COPD), pathological diagnosis, tumor location, TNM stage, radiation dose, and concurrent chemoradiotherapy lack thereof. In addition, the lung dosimetric parameters involved in this study included V5-V40 (relative volume of total lung treated with $\geq 5-40 \mathrm{~Gy}$ ) and MLD. In short, the parameters mentioned above were used to establish a comprehensive nomogram after univariate analysis or least absolute shrinkage selection operator (LASSO) feature selection.

The Eclipse Treatment Planning System (Varian Medical Systems, Palo Alto, CA, Version 13.5.35) was adopted for RT planning design. IMRT adopts a fixed-field, static intensity modulation technique, and 5-7 fields of coplanar irradiation are uniformly divided according to the specific situation in each case. The required target parameters are then set, and the dose distribution is obtained by inverse calculation of the treatment planning system. The dose distribution is then graded (stratified), and each field is decomposed into a series of subfields. IMRT does not include sIMRT or volumetric intensity-modulated arc therapy (VMAT). The target area includes tumor volume (GTV), including CT imaging of visible esophageal tumors and positive lymph nodes. The clinical target volume (CTV) refers to the upper and lower expansion of the esophageal tumor by $3 \mathrm{~cm}$ and $6 \mathrm{~mm}$ around the tumor and related lymphatic drainage area. The planned target volume (PTV) is formed by CTV extending $8 \mathrm{~mm}$ outward. IMRT was administered by a Varian Linac Accelerator with a 6-MVX ray and 95\% PTV, and radiation doses of 50-66 Gy (median dose of $60 \mathrm{~Gy}$ ) and 1.8-2.0 Gy/fraction 5 times/week were prescribed.

Normal tissue constraints were prioritized in the following order for treatment planning purposes: maximum spinal cord dose of $45 \mathrm{~Gy}$, relative volume of total lung treated with $\geq 5 \mathrm{~Gy}$ (V5) $\leq 60 \%$, relative volume of total lung treated with $\geq 20 \mathrm{~Gy}$ (V20) $\leq 28 \%$, MLD $\leq 20 \mathrm{~Gy}$, relative volume of the heart treated with $\geq 30$ Gy $(\mathrm{V} 30) \leq 40 \%$, and relative volume of the heart treated with $\geq 40$ Gy (V40) $\leq 30 \%$.

\section{Follow-up and Evaluation of RP}

Follow-up items included chest CT, physical examination, and clinical symptoms. Patients were evaluated weekly during RT, followed up at 1 month after completion of the initial treatment, and then followed up every 2-3 months until at least 6 months after the end of RT. The grading of RP was confirmed by two senior oncologists and one radiologist. The National Cancer Institute Common Terminology Criteria for Adverse Events 4.03 (CTCAE 4.03) was used to evaluate the degree of RP. In the present study, grade $\geq 2$ was used as the cutoff for diagnosing RP.

\section{CBCT Scanning Method and Image Acquisition}

Using the on-board imager (OBI) system mounted on the Varian Trilogy medical linear accelerator, the hardware portion included a diagnostic $(\mathrm{kV})$ level X-ray source (KVS) and an amorphous silicon flat-panel $\mathrm{kV}$ detector (KVD). The CBCT image was obtained by rotating the frame at an angle. This is a slow CBCT acquisition setting. The acquisition time is $67 \mathrm{~s}$, and the patient keeps breathing evenly during this process. Standard body scan conditions were voltage $(125 \mathrm{kVp})$, current (80 $\mathrm{mA})$, exposure time (13 ms), exposure $(680 \mathrm{mAs})$, rotation angle $\left(178^{\circ}-182^{\circ}\right)$, pixel matrix size (384×384), field of view (FOV , $45 \times 18 \mathrm{~cm})$, slice thickness $(2.5 \mathrm{~mm})$, and fan-beam type (half-fan). Among fan-beam types, the half-fan mode was used for the image acquisition of lung tissue structures larger than $24 \mathrm{~cm}$. In this study, lung CBCT image acquisition was carried out in three different periods, and then the images were imported into 3D Slicer (version 4.10.2; http://www.slicer.org) in a DICOM format to extract and analyze the radiomics features. It should be noted that these three different periods were artificially divided according to the experimental design and corresponded to the early stages: the third, fourth, and fifth weeks of RT (PTV prescription dose range of EC: 18-22 Gy, 27-32 Gy, and 36-44 Gy).

\section{Image Segmentation and Feature Extraction}

Images from both lungs were segmented by a semiautomatic segmentation method $(16,17)$ based on a threshold-based algorithm. The specific steps are as follows: First, the background was removed to obtain the internal region of the chest. Second, the appropriate threshold was found to segment the lung and the tissues outside the lung contour to the greatest extent. Finally, the manual segmentation method (18) was used to erase the extra parts outside the large trachea and lung parenchyma to obtain both lungs as the ROI. Image segmentation was performed by an experienced radiologist and then verified by a senior radiologist. All features were extracted by using the radiomics plug-in in 3D Slicer. A total of 851 radiomics features were extracted, including 13 morphological features, 18 histogram features, 74 original texture features, and 746 high-order features (wavelet transform features).

\section{Radiomics Feature Selection and Radiomics Signature Construction}

First, the extracted radiomics features were preprocessed. Based on the Spearman rank correlation test, the features with correlations greater than 0.9 and multicollinearity were deleted, and independent features were preliminarily screened. Meanwhile, based on the Mann-Whitney $U$ test, the characteristics with significant differences between the RP ( $\geq 2$ grade) and non-RP ( $<2$ grade) groups were screened out. Finally, the LASSO method (19) was used to select the final features, and the RP prediction model of rad-score was constructed based on logistic regression analysis. The LASSO method minimizes the sum of squared residuals by using the case in which the sum of the absolute values of the coefficients is less than the tuning parameter $(\lambda)$. To prevent overfitting of the model, (20-22) during model building, features are selected by constantly adjusting $\lambda$. With the increasing penalty, more regression coefficients are reduced to zero, $(23,24)$ and then the remaining nonzero coefficient is selected. The nonzero coefficient of the selected features is the rad-score. Each patient's rad-score is calculated as a linear combination of selected features that have their own coefficient weighting. 
In this study, 50 iterations of 10 -fold nested cross-validation were utilized, similarly to $\mathrm{Xu}$ et al. (25). Random sampling was conducted in an attempt to balance the class distributions within the cross-validation partitions. The cross-validation loop provides a profile of model performance. It serves to estimate how well the LASSO applied to a given set of candidate predictors may generalize to other data sets. Model performance was assessed by computing the area under the curve (AUC) for each constructed model on a test partition. The inner crossvalidation loop was applied to determine the optimal value for $\lambda$ such that the resulting model was guarded against overfitting. The value of $\lambda$ for each cross-validation partition was selected by determining the value that produced the most regularized model such that the AUC was within one standard error of the maximum (26). The use of 50 resampled iterations with 10 -fold nested crossvalidation constructs 500 models used to generate a distribution of AUC values to estimate how well model construction with LASSO generalizes to other data sets.

\section{Construction and Validation of the Nomogram}

First, the prediction efficiency of the three different periods was compared, and then the best period was selected. Second, 96 patients were divided into the RP (39 cases) and non-RP (57 cases) groups, and 16 clinical features and dosimetric parameters were collected. The best clinical features and dosimetric parameters were determined by LASSO feature selection. Finally, a comprehensive nomogram was established. The receiver operating characteristic (ROC) curve was used to evaluate the prediction capacity of the model. The calibration curve was used to determine whether the predicted and observed probabilities for RP were in concordance. The decision curve was performed to evaluate the clinical benefit ratio of the nomogram.

This research process can be divided into four parts: image acquisition, ROI segmentation, feature extraction, and radiomics model construction as shown in Figure 1.

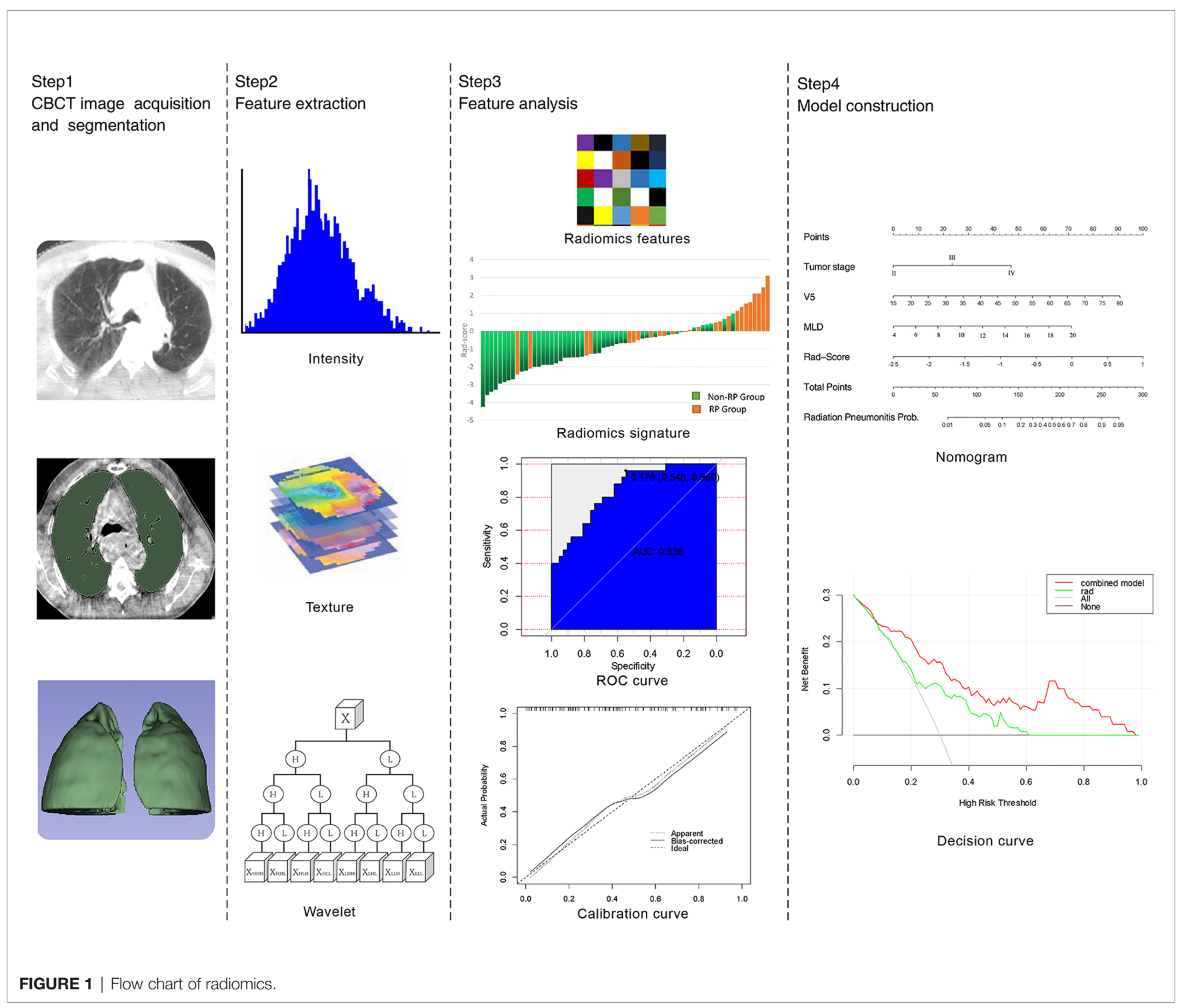




\section{Statistical Analysis}

All statistical analyses were based on SPSS 20.0 (IBM, Armonk, NY, USA) or R software (R Foundation for Statistical Computing, Vienna, Austria, https://www.R-project.org/). The $\chi^{2}$ test or Fisher exact probability test was used to classify data between the two groups. Two independent-sample $t$ tests were used for counting data (continuous data). The Mann-Whitney $U$ test was used to compare the differences in clinical features between the primary and validation cohorts. The model was evaluated with respect to sensitivity, specificity, ROC curve, and 95\% confidence interval (CI). $P$ values $\leq 0.05$ were considered statistically significant.

\section{RESULTS}

\section{Analysis of Clinical Features and Dosimetric Parameters Associated With RP}

The 96 patients were divided into RP (39 cases) and non-RP (57 cases) groups, and 9 clinical features and 7 dosimetric parameters that might be related to the occurrence of RP were included. Univariate analysis showed that tumor stage was correlated with $\geq 2$ grade $\mathrm{RP}\left(\chi^{2}=2.650, P=0.008\right)$, and other factors, including age, sex, concurrent chemoradiotherapy or lack thereof, COPD status, smoking status, and RT dose, showed no significant differences between the two groups (all Ps > 0.05). V5, V10, V15, V20, V30, and MLD of both lungs were associated with the occurrence of grade $\geq 2 \mathrm{RP}$ (all Ps $<0.05$ ). The characteristics of the enrolled population are listed in Tables $\mathbf{1}$ and $\mathbf{2}$.

There were no significant differences in age, sex, tumor stage, V5, and MLD between the primary group and the validation group, which indicates that the groupings were reasonable (all $P$ s $>0.05$ ) as shown in Table 3. Seven factors (tumor stage, V5, V10, V15, V20, V30, and MLD) remained after univariate analysis. The LASSO feature selection method was used to screen these seven factors, and three potential factors (V5, MLD, and tumor stage) remained as shown in Figures 2A, B. The AUC values of prediction efficiency for V5, MLD, and tumor stage were 0.698 , 0.685 , and 0.662 , respectively. To observe the overall predictive performance of V5, MLD, and tumor stage, we established a full clinical-dosimetric feature combined model. The AUC value of the combined model was 0.764 as shown in Figure 2C.

\section{Radiomics Feature Extraction/Selection at Different Periods and Radiomics Signature Building}

In the first period (PTV dose: 18-22 Gy), a total of 851 radiomics features were extracted from the patients. First, correlations greater than 0.9 features were deleted, resulting in a total of 220 features remaining. Second, linear features were removed, and 96 features remained. Then, 21 features remained after using the rank-sum test. Finally, the remaining two features after LASSO selection were used to build the radiomics model as shown in Figures 3A, B. The two features are original first-order skewness and original GLSZMsmall area emphasis. The model was built as follows: Rad-score = -0.924 e+00 $\times$ Skewness - 7.047 e+00 $\times$ Small Area Emphasis +
TABLE 1 | Univariate analysis of baseline clinical features of patients and RP.

\begin{tabular}{|c|c|c|c|c|c|}
\hline \multirow[t]{2}{*}{ Factor } & \multirow[t]{2}{*}{$N$} & \multicolumn{2}{|c|}{$\mathbf{R P}$} & \multirow[t]{2}{*}{$\chi^{2}$ value } & \multirow[t]{2}{*}{$P$ value } \\
\hline & & $<2$ grade & $\geq 2$ grade & & \\
\hline Sex & 96 & 57 & 39 & 2.767 & 0.096 \\
\hline Male & 81 & 51 & 30 & & \\
\hline Female & 15 & 6 & 9 & & \\
\hline Age (years) & 96 & & & 1.619 & 0.203 \\
\hline$<60$ & 21 & 15 & 6 & & \\
\hline$\geq 60$ & 75 & 42 & 33 & & \\
\hline Stage & & & & 2.650 & 0.008 \\
\hline II & 19 & 15 & 4 & & \\
\hline III & 48 & 30 & 18 & & \\
\hline IV & 29 & 12 & 17 & & \\
\hline Smoking history & 96 & & & 0.198 & 0.656 \\
\hline No & 54 & 31 & 23 & & \\
\hline Yes & 42 & 26 & 16 & & \\
\hline COPD & 96 & & & 1.436 & 0.231 \\
\hline No & 81 & 46 & 35 & & \\
\hline Yes & 15 & 11 & 4 & & \\
\hline Diabetes & 96 & & & 0.318 & 0.573 \\
\hline No & 88 & 53 & 35 & & \\
\hline Yes & 8 & 4 & 4 & & \\
\hline Hypertension & 96 & & & 0.606 & 0.436 \\
\hline No & 83 & 48 & 35 & & \\
\hline Yes & 13 & 9 & 4 & & \\
\hline Concurrent & 96 & & & & \\
\hline \multicolumn{6}{|l|}{ Chemotherapy } \\
\hline No & 71 & 41 & 30 & 0.300 & 0.584 \\
\hline Yes & 25 & 16 & 9 & & \\
\hline Delivered & 96 & & & 1.867 & 0.172 \\
\hline \multicolumn{6}{|l|}{ Dose (Gy) } \\
\hline$<60$ & 45 & 30 & 15 & & \\
\hline$\geq 60$ & 51 & 27 & 24 & & \\
\hline
\end{tabular}

COPD, chronic obstructive lung disease.

TABLE 2 | Single factor analysis of DVH and RP.

\begin{tabular}{lcccc}
\hline \multirow{2}{*}{ Lung DVH } & \multicolumn{2}{c}{$\mathbf{R P}$} & $\boldsymbol{P}$ value & $\boldsymbol{\chi}^{\mathbf{2}}$ value \\
\cline { 2 - 3 } & \multicolumn{2}{c}{ grade } & $\mathbf{2}$ grade & \\
\hline V5 & $48.95 \pm 10.56$ & $59.39 \pm 10.00$ & 0.00 & -4.91 \\
V10 & $33.64 \pm 7.70$ & $40.92 \pm 7.95$ & 0.00 & -4.46 \\
V15 & $25.34 \pm 6.52$ & $30.77 \pm 6.96$ & 0.00 & -3.85 \\
V20 & $18.81 \pm 5.47$ & $22.47 \pm 4.82$ & 0.00 & -3.47 \\
V30 & $9.61 \pm 4.40$ & $12.16 \pm 5.00$ & 0.01 & -2.58 \\
V40 & $3.80 \pm 2.49$ & $4.58 \pm 3.24$ & 0.21 & -1.25 \\
MLD (cGy) & $1016.47 \pm 218.82$ & $1260.87 \pm 267.38$ & 0.00 & -4.72 \\
\hline
\end{tabular}

MLD, mean lung dose; V5, V10, V15, V20, V30, V40 = relative volume of total lung treated with $\geq 5,10,15,20,30$, and $40 \mathrm{~Gy}$.

TABLE 3 | Comparison of sex, age, tumor stage, V5, and MLD between the primary and the verification cohort.

\begin{tabular}{lcccc}
\hline Factor & Primary cohort & Verification cohort & $\chi^{\mathbf{2}}$ value & $\boldsymbol{P}$ value \\
\hline Age (years) & $65.33 \pm 9.37$ & $68.62 \pm 8.89$ & -1.64 & 0.11 \\
Sex (N) & 67 & 29 & 0.11 & 0.75 \\
$\quad$ Male & 56 & 25 & & \\
$\quad$ Female & 11 & 4 & & \\
Stage & 13 & 6 & 3.54 & 0.17 \\
II & 30 & 18 & & \\
III & 24 & 5 & & \\
IV & $52.35 \pm 11.27$ & $55.14 \pm 12.01$ & -1.07 & 0.29 \\
V5 & $11.06 \pm 2.61$ & $11.38 \pm 2.85$ & -0.52 & 0.61 \\
MLD (Gy) & & & &
\end{tabular}


A

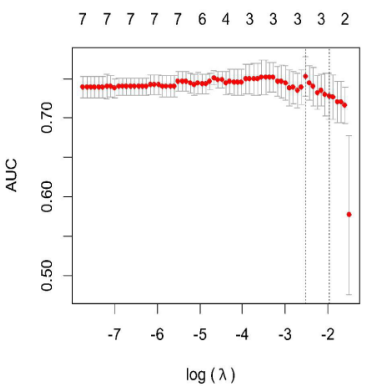

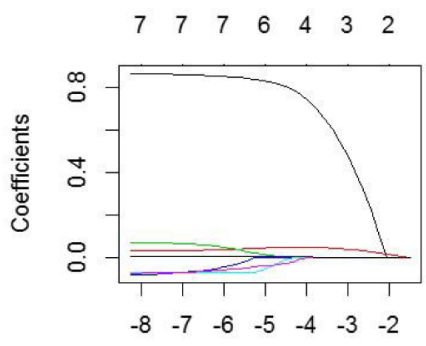

$\log (\lambda)$
C

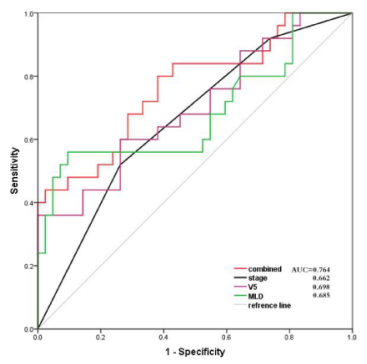

FIGURE 2 | LASSO characteristic selection of clinical features and dosimetric parameters (A, B). ROC curve of V5, MLD, tumor stage, and combined model (C).

A

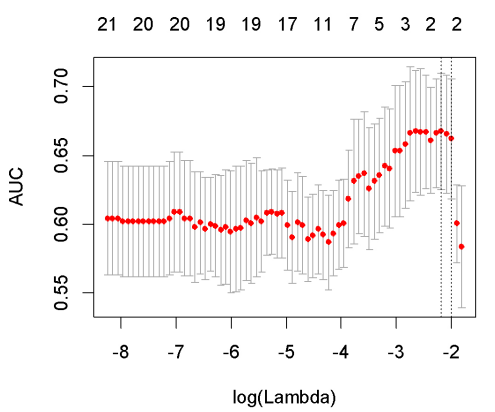

B

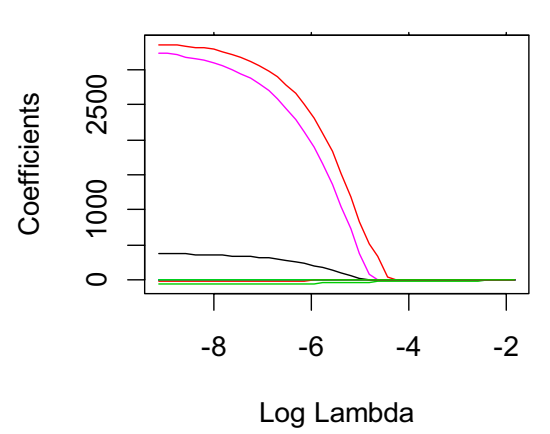

FIGURE 3 | Feature screening of radiomics in the first period. By adjusting the different penalty parameter $(\lambda)$ to obtain a high-performance model, the radiomics characteristics with the highest predictive performance were obtained. Radiomics feature convergence diagram (A). Each curve represents the trajectory of the coefficient of each independent variable (B).

4.5329. Rad-scores for each patient in the primary cohort and validation cohort are shown in Figures 4A, B.

In the second period (PTV dose: 27-32 Gy), a total of 851 radiomics features were extracted from the patients. First, correlations greater than 0.9 features were deleted, resulting in a total of 222 features remaining. Second, linear features were removed, and 96 features remained. Then, 10 features remained after using the rank-sum test. Finally, the remaining five features (voxel volume, smallest axis length, small dependence low gray-level emphasis, large area low gray-level emphasis, and busyness) after LASSO selection were used to build the radiomics model. The model was built as follows: Rad-score $=-1.996$ e- $07 \times$ voxel volume -4.036 e- $03 \times$ smallest axis length +5.376 e+ $01 \times$ small dependence low gray-level emphasis +1.718 e-07×large area low gray-level emphasis 2.473 e-04×busyness +1.041 e+00.

In the third period (PTV dose: 36-44 Gy), a total of 851 radiomics features were extracted from the patients. First, correlations greater than 0.9 features were deleted, resulting in a total of 220 features remaining. Second, linear features were removed, and 96 features remained. Then, 43 features remained after using the rank-sum test. Finally, the remaining six features (gray-level nonuniformity, small dependence low gray-level emphasis, cluster shape, uniformity, entropy, and size zone nonuniformity) after LASSO selection were used to build the radiomics model. The model was built as follows: Rad-score = +4.680 e-07×gray-level nonuniformity +1.087 e+01×small dependence low gray-level emphasis - 7.913 e-04×cluster shape +1.401 e+00×uniformity +1.406 e+00 $\times$ entropy -2.207 e- $05 \times$ size zone nonuniformity $-4.776 \mathrm{e}+00$.

\section{Validation of Radiomics Signature at Different Periods}

In the first period, the predictive efficacy of the model was as follows: In the primary cohort, the AUC was 0.700 (95\% CI $0.568-0.832$ ), the sensitivity was $61.5 \%$, and the specificity was $75.0 \%$. In the validation cohort, the AUC was 0.765 (95\% CI $0.588-0.941$ ), the sensitivity was $84.6 \%$, and the specificity was $64.7 \%$ as shown in Table 4 and Figures 5A, B.

In the second period, the predictive efficacy of the model was as follows: In the primary cohort, the AUC was 0.663 (95\% CI $0.530-0.797$ ), the sensitivity was $90.6 \%$, and the specificity was 


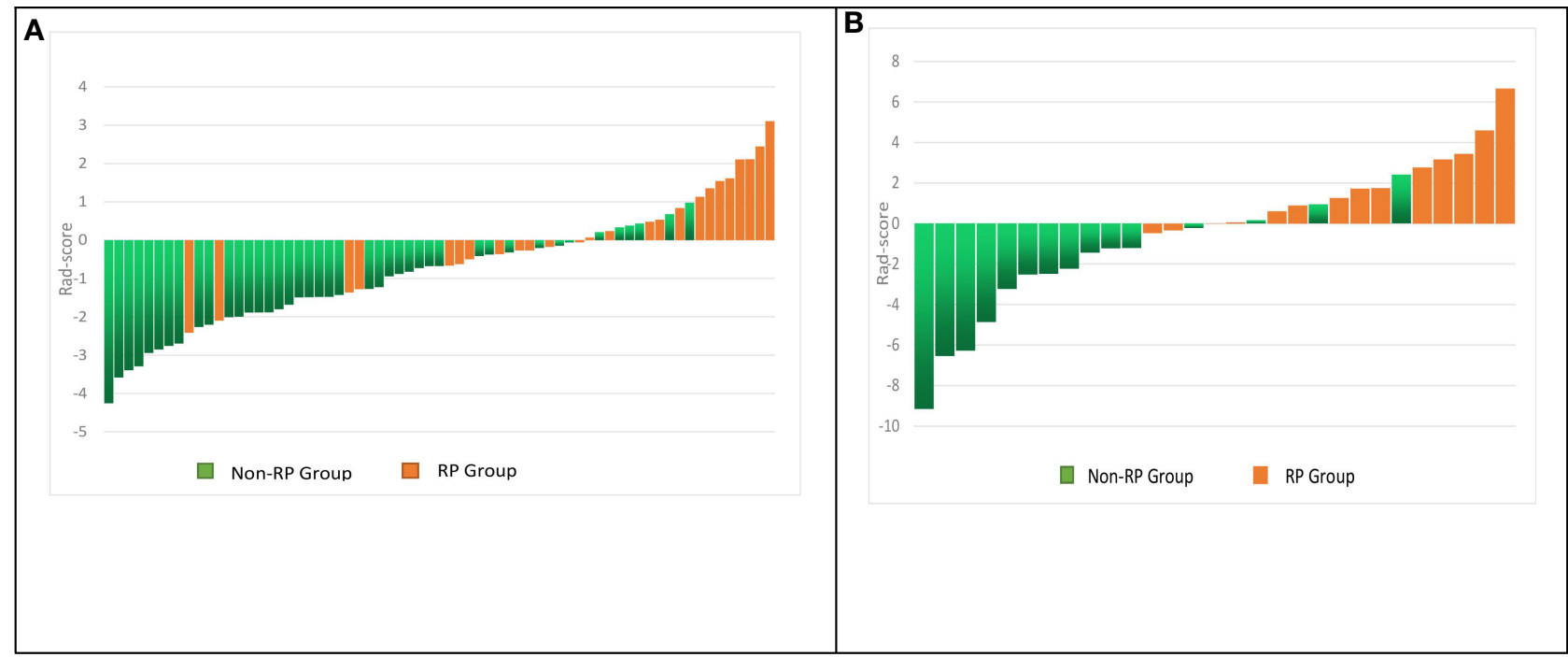

FIGURE 4 | Rad-score for each patient in the primary and validation cohorts. Green bars show scores for patients without RP, and orange bars show scores for those with RP (A, B).

TABLE 4 | ROC curve parameters of the radiomics model and nomogram.

\begin{tabular}{|c|c|c|c|c|c|c|c|c|}
\hline Classification & \multicolumn{4}{|c|}{ Primary cohort } & \multicolumn{4}{|c|}{ Validation cohort } \\
\hline First period & 0.700 & $0.568-0.832$ & $61.5 \%$ & $75.0 \%$ & 0.765 & $0.588-0.941$ & $84.6 \%$ & $64.7 \%$ \\
\hline Third period & 0.699 & $0.573-0.826$ & $66.7 \%$ & $70.3 \%$ & 0.756 & $0.561-0.950$ & $66.7 \%$ & $80.0 \%$ \\
\hline Nomogram & 0.836 & $0.700-0.918$ & $96.0 \%$ & $54.8 \%$ & 0.905 & $0.799-1.000$ & $92.9 \%$ & $73.3 \%$ \\
\hline
\end{tabular}

A

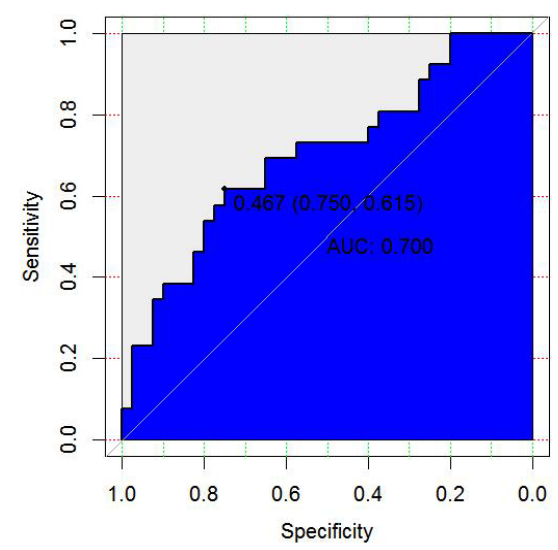

B

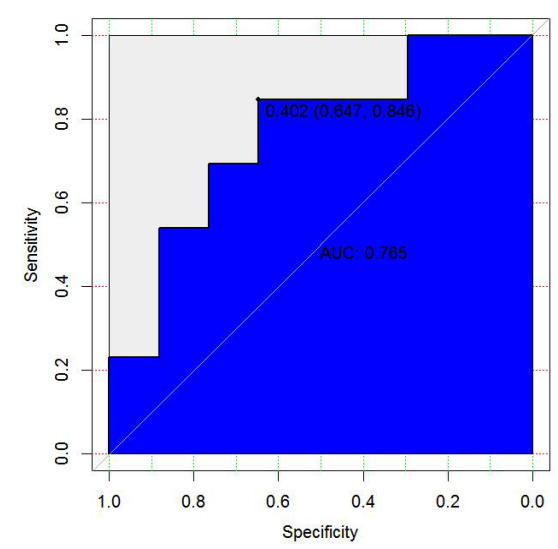

FIGURE 5 | ROC curve of radiomics features in the first period of RT (A, B). 
42.9\%. In the validation cohort, the AUC was 0.604 (95\% CI $0.356-0.851)$, the sensitivity was $85.7 \%$, and the specificity $50.0 \%$.

In the third period, the predictive efficacy of the model was as follows: In the primary cohort, the AUC was $0.699(95 \%$ CI $0.573-0.826$ ), the sensitivity was $66.7 \%$, and the specificity was $70.3 \%$. In the validation cohort, the AUC was 0.756 (95\% CI $0.561-0.950)$, the sensitivity was $66.7 \%$, and the specificity was $80.0 \%$ as shown in Table 4 .

By comparing the prediction efficiency of the AUC in three periods, it is obvious that the prediction efficiency in the first period is better than those in the second and third periods in both the primary and validation cohorts. To reflect the importance of the early prediction of RP in clinical practice, the first-period rad-score and three essential features (V5, MLD, and tumor stage) were used to establish a comprehensive nomogram model.

\section{The Incremental Value of the Radiomics Signature When Added to the Comprehensive Nomogram}

The AUC values of dosimetric parameters (V5, MLD) and clinical features (tumor stage) were 0.698, 0.685, and 0.662, respectively. The AUC values of the full clinical-dosimetric feature combined model was 0.764. In addition, the AUC values of the radiomics signature at three different periods were $0.700,0.663$, and 0.699 , respectively (primary cohort). It can be seen that the single clinical features, dosimetric parameters, or full clinical-dosimetric combined model are not ideal in predicting the risk of RP. To this end, we created a comprehensive nomogram that integrates dosimetric parameters and clinical features with the radiomics signature from the first period. The results show that, in the primary cohort, the AUC of our nomogram was 0.836 (95\% CI: $0.700-0.918$ ), and in the validation cohort, the AUC was 0.905 (95\% CI: 0.799-1.000) as shown in Table 4 and Figures 6B, C. There is no doubt that the comprehensive nomogram, incorporating radiomics features, significantly improves the ability of conventional dosimetric parameters and clinical features to predict the risk of RP. The graphical form of the nomogram is shown in Figure 6A. More importantly, the calibration curve is produced as shown in Figure 6D. The diagonal dotted line represents an ideal evaluation, and the other two lines next to it represent the performance of the nomogram. A closer fit to the diagonal dotted line indicates a better evaluation. In summary, this calibration curve shows favorable consistency between the predicted RP and the actual observation.

\section{How to Make Clinical Decisions}

The clinical decision curve analysis of the nomogram is shown in Figure 6E, which shows the patient's benefits when the physician makes the judgment. It shows that, if the probability of the domain value is $10 \%$, the benefit of using the nomogram to predict the efficacy of RP is higher than that of radiomics features or other parameters alone. In short, this decision curve exhibits satisfactory positive net benefits of the nomogram at the threshold probabilities.

\section{DISCUSSION}

A single index based on lung dosimetric parameters is not the "gold standard" to judge the occurrence of PR risk; however, radiomics can extract image data to characterize the standard tissue structure, including typical lung structures. They may produce clinically relevant improvements in predicting treatment-related toxicities (13). This makes up for the deficiency of dose-volume parameter prediction to a great extent. Some previous studies, $(12,13)$ respectively, report the relationship between the changes in some second- or higherorder eigenvalues of lung cancer after and before RT and the occurrence of RP. Unfortunately, due to the limitations of detection techniques or other factors, it is not possible to establish predictive models for clinical practice. In this study, we used an automated computer extraction algorithm and digital quantitative analysis technology to obtain high-quality information to comprehensively evaluate various characteristics of tumor and normal tissue responses (14, 27). More importantly, we constructed a comprehensive nomogram model based on CBCT radiomics features in combination with clinical features and dosimetric parameters to accurately predict RP in EC patients treated with RT. To the best of our knowledge, this is the first study of the early prediction of RP by using IGRT to obtain CBCT imaging information in different periods of RT. Importantly, this comprehensive nomogram model is superior to single clinical features and lung dosimetric parameters in $\mathrm{RP}$ prediction.

We selected CBCT images from three different periods and extracted the radiomics features. The primary purpose was to find the first radiomics features that can independently predict RP; however, after selecting the radiomics features in different periods, it is found that each period has its own independent set of feature parameters related to RP. We believe that, in addition to the influence of radiation dose factors, whether these characteristics vary with changes in the RT process is still uncertain. It is gratifying that we found the best prediction of $\mathrm{RP}$ to be in the first period of radiomics characteristics. Two important features can be found in the early stage of low-dose RT of lung tissue: Although this may differ from our initial expectation of the experimental results, the results are fascinating. This result is similar to the findings of Cunliffe et al. (12) and Jenkins et al (28). They found that AUC values in low- and medium-dose areas of the lung were different between RP and non-RP patients even though these AUC values appeared in areas with lower visible changes. These first radiomics features may be able to be used to explain or screen out those susceptible to RP due to intrinsic genetic mutations.

In regard to the susceptible population of RP, we must devote attention to the sensitivity of lung tissue to RT. At present, the radiosensitivity of lung tissue has been reported $(29,30)$, and it is considered to be a potential influencing factor for RP occurrence. This difference in the sensitivity of lung tissue to radiation constitutes our different understanding of the probability of RP. In two groups of patients with different radiosensitivity of lung tissue, we cannot judge the probability of RP by standard clinical features and lung dosimetric parameters. However, 
Points

Tumor stage

V5

MLD

Rad-score

Total Points

RP probability

B

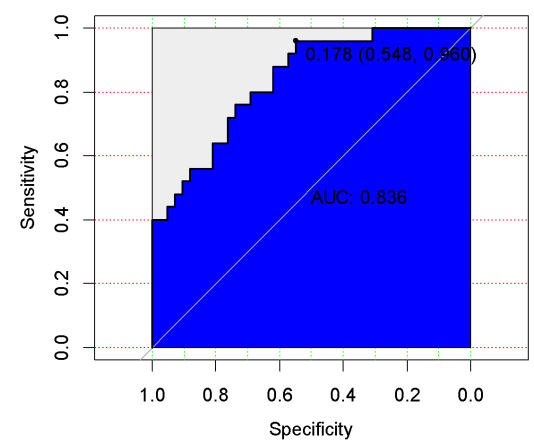

Nomogram

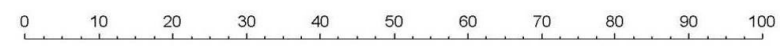

III

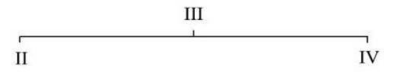

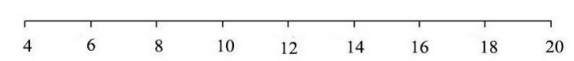

\begin{tabular}{rrrrrrrr}
\hline-2.5 & -2 & -1.5 & -1 & -0.5 & 0 & 0.5 & 1
\end{tabular}

\begin{tabular}{llllll}
\hline & 1 & 1 & 1 & 1 & 1 \\
\hline & 50 & 100 & 200 & 300
\end{tabular}

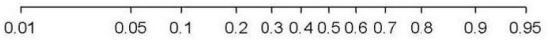

C
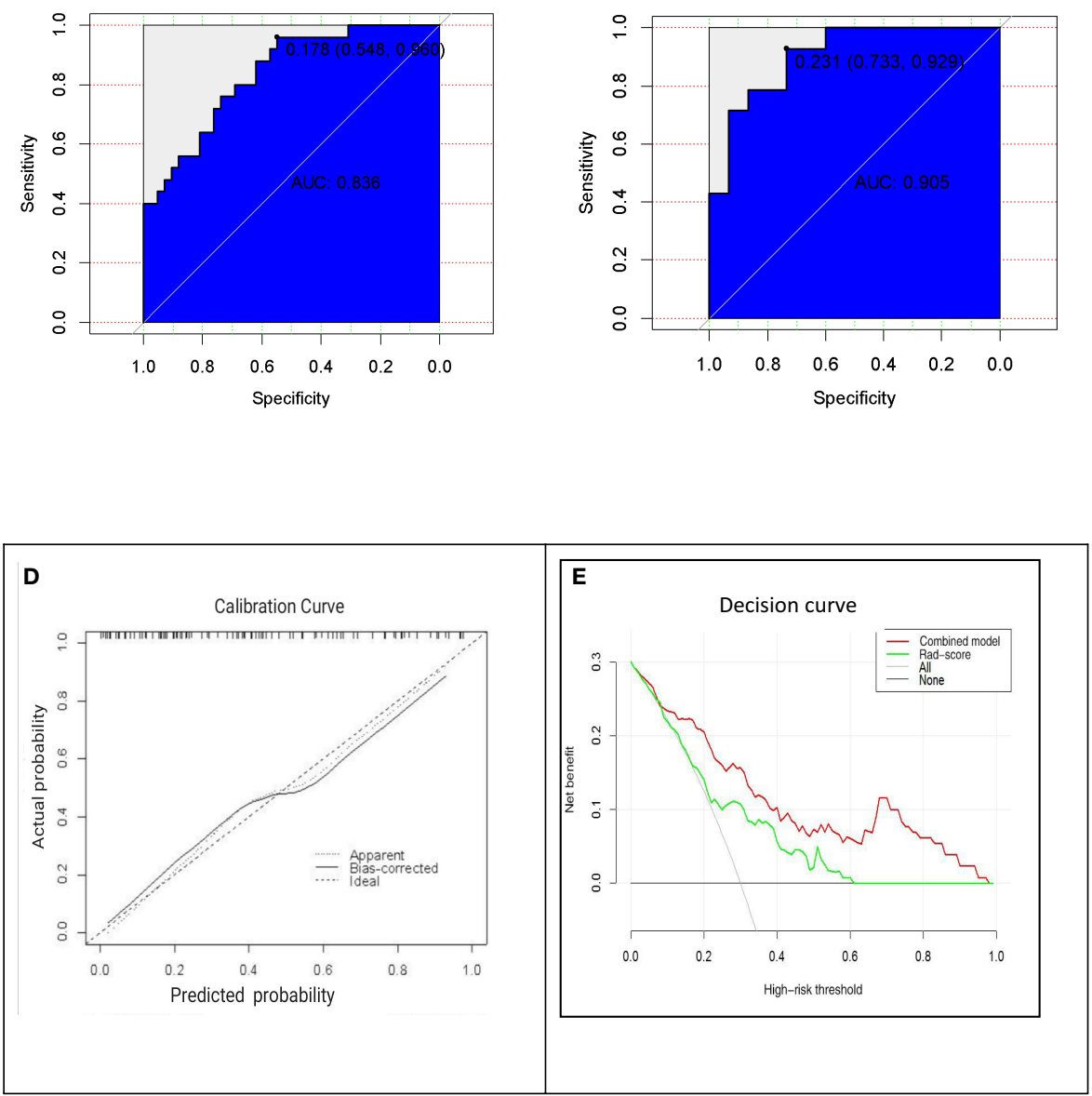

FIGURE 6 | (A) The comprehensive nomogram incorporates V5, MLD, tumor stage, and rad-score (the first period) to predict the risk of RP in EC patients. V5: relative volume of total lung treated with $\geq 5$ Gy; MLD: mean lung dose. (B, C) ROC curves of the comprehensive nomogram in the primary and validation cohorts. (D) Calibration curves of the comprehensive nomogram with the addition of V5, MLD, tumor stage, and radiomics features. The diagonal dotted line represents an ideal evaluation, and the other two lines next to it represent the performance of the nomogram. A closer fit to the diagonal dotted line indicates a better evaluation. (E) Decision curves of the radiomics features model and the combination model (comprehensive nomogram) predicting RP. The $y$-axis represents the net benefit. The red curve represents the comprehensive nomogram, and the green line represents the radiomics features model. The horizontal black line indicates that the assumption is valid. The oblique gray line indicates that the assumption is invalid. 
radiomics can analyze the data by extracting features from CT images of the lung, thus providing a powerful method for the noninvasive description of lung tissue radiosensitivity. This may be why the radiomics features are superior to the clinical features and dosimetric parameters in current studies. In this study, this advantage in AUC value, sensitivity, and specificity performance is not particularly good, but through our research analysis, radiomics features of RP risk prediction cannot be ignored.

The successful establishment of the prediction model is based on the standardization of data collection and the rationalization of data processing. First, we should consider that the feature extraction data are affected by CT parameters (31) because the CT features may be different under different image-acquisition conditions. In this study, based on the CBCT of the Varian accelerators in our center, these devices have the same tube voltage, tube current, exposure time, exposure amount, and pixel matrix size, which can help control for the differences between the scanners and acquisition parameters. Second, to develop the radiomics signature, a total of 851 candidate features were reduced to a set of only a few potential descriptors by using the LASSO logistic regression model to realize feature selection by constantly adjusting the regularization parameter $\lambda$ to make the weight coefficient of the feature approach zero. The LASSO (20) logistic regression model is suitable for analyzing large sets of radiomics features with a relatively small sample size, and it is designed to avoid overfitting high-dimensional data $(21,32)$. At the same time, the LASSO logistic regression model allows the radiomics signature to be constructed by combining the selected features, so it allows the model to more easily identify the most closely related features in patients with RP. Finally, the nested cross-validation method (25) was used for internal validation to improve the accuracy of the model.

It should be noted that the difference in the irradiation mode (3-D conformal radiation therapy and IMRT) affects the potential dose distribution of the lung, which may affect the selection of clinical features and dosimetric parameters as risk characteristics of RP. This can be quickly confirmed by comparing Tucker et al. (33) and Shane et al. (13) where, in the former, $75 \%$ received $3-\mathrm{D}$ conformal radiation therapy, and the latter $83 \%$ received IMRT. Therefore, it seems complicated to establish a general model with good discriminant performance under different technical conditions.

The clinical factors (age, tumor stage, KPS score, chronic lung disease, diabetes, chemotherapy lack thereof) and lung dosimetric parameters (V5, V10, V20, MLD) related to RP are reported in previous studies. To provide better help for the oncologist, we designed a clinical nomogram to combine the above available RP risk factors with radiomics features. Therefore, we aim to establish a combined model, maximizing clinical utility and accuracy of prediction ability, and so the initial experimental design was not expected to rely solely on the radiomics model as the final prediction model. Of course, judging from the AUC value, sensitivity, and specificity of the radiomics model in each period of RT, these characteristics alone are not perfect in predicting RP. Dose-volume histogram (DVH) metrics have been extensively observed and reported to be correlated with RP despite the current data and research reports not being sufficient to provide specific and safe standard doses (34). Chargari et al. (35) find that V5 is a risk factor for acute or chronic lung toxicity. Cho et al. (6) find that MLD is the most related factor that predicts RP rather than V5, V10, or V20. Some clinical features have emerged as important risk factors contributing to RP progression. Some studies show that smoking is related to the severity of $\operatorname{RP}(36,37)$. Takeda et al. (38) and Kimura et al. (39) report that COPD is a significant risk factor for RP in patients with EC after RT. In this study, we find that smoking status, COPD, and concurrent chemoradiotherapy are not correlated with the incidence of RP, and so these factors are not included in our combined model, but this does not mean that they are not important. After LASSO logistic regression analysis, several significant variables, including V5, MLD, and tumor stage, were integrated into the nomogram to predict PR. The results were as follows: clinical-dose characteristic model (AUC values: V5 $=0.698, \mathrm{MLD}=0.685$, tumor stage $=0.662$ ), radiomics model (primary cohort AUC 0.700 , validation cohort AUC 0.765), and nomogram (primary cohort AUC 0.836, validation cohort AUC 0.905). The nomogram demonstrates a better ability to predict RP than the other models.

How to use this information in the treatment plan or alternative program to help clinicians is our greatest concern. Fortunately, the goal of radiomics is to develop a decisionmaking tool that meets the needs of clinicians. This is because such a tool could combine radiomics features with other patient characteristics to improve the capability of the decision support model $(15,40)$. We show that radiomics features complement clinical features and lung dosimetric parameters, helping to provide better predictive ability for RP. The clinical decision curve of this nomogram shows that the effectiveness of the nomogram in predicting $\mathrm{RP}$ is higher than that of using radiomic characteristics or other parameters alone. In short, under the threshold probability, the decision curve exhibits a satisfactory positive net benefit of the nomogram.

Our results demonstrate the potential value of radiomics techniques in the risk prediction of RP patients. If more clinical variables are included in the nomogram, there will be more room for future development of this model, and the resulting prediction effect will be better. A recent study (41) by another of our teams found that subjective global assessment score (SGA), pulmonary fibrosis score (PFS), planning target volume/total lung volume (PTV/LV), MLD, and ratio of change regarding systemic immune inflammation index at 4 weeks ( $4 \mathrm{w}$ SII) were potential valuable markers in predicting severe acute radiation pneumonitis (SARP). Subsequently, the team developed a nomogram and corresponding risk classification system with superior prediction ability for SARP. In the next step, we will consider combining the research results of this team with radiomics to establish a new RP prediction model for better clinical application.

Although our study has many strengths, several limitations should be addressed here. First, the sample size is small, which can lead to the inability to apply nonlinear classifiers, such as neural networks $(42,43)$. Because a nonlinear classifier uses a more extensive data set, it is beneficial to improve the accuracy of the RP model. Second, our analysis does not account for two-way 
or higher-order interactions between features. If interactions between features had been identified, the interaction terms that were most strongly associated with the outcome interactions would have been selected when we constructed the radiomics signature, and this could have improved performance. However, uncovering the interactions of multiple attributes is a challenging problem. Third, we used a validation cohort that was drawn from the same institution as the primary cohort, which prevented us from investigating the generalizability of the results to other institutions and settings. In addition, there is a lack of sufficient external data validation. Fourth, selection bias occurred when strict criteria were used, and this may affect the model training. For instance, all patients are middle thoracic EC patients, which limits the application of this method to patients with cervical, upper, and lower thoracic segment EC radiotherapy. Also, all patients experienced uniform CBCT imaging scanners and parameters, which does not guarantee the reproducibility and stability of radiomics features under other conditions. In the future, we should conduct a prospective, multicenter, largecohort study to further develop and validate nomograms in terms of predicting RP.

As a future study, we will add different types of patients, including those with different EC locations (cervical, upper thoracic, lower thoracic segments) and different RT techniques (3DCRT, TOMO, VMAT). We will also include more laboratory indicators that may reflect RP, such as inflammatory indexes and immune inflammatory indexes. In terms of basic research, we should also improve the model of radiomics, especially the combination of radiomics and genomics. The former focuses on medical imaging of the normal tissues or tumors and performs diagnosis and prognosis based on quantitative imaging features, and the latter discovers and notes the gene sequences to study the function and structure of genomes of the diseases. Besides this, if we can combine available radiation metabolomics (44) with functional CT $(45,46)$ radiomics features, it may help us understand the differences in radiation sensitivity and tissue cell metabolism in order to establish a more robust prediction model. Therefore, it can be predicted that the combination of multiple omics will be the best plan for the future

\section{REFERENCES}

1. Bray F, Ferlay J, Soerjomataram I, Siegel RL, Torre LA, Jemal A. Global cancer statistics 2018: GLOBOCAN estimates of incidence and mortality worldwide for 36 cancers in 185 countries. CA Cancer J Clin (2018) 68(6):394-424. doi: $10.3322 /$ caac. 21492

2. Ajani JA, D'Amico TA, Bentrem DJ, Chao J, Corvera C, Das P, et al. Esophageal and Esophagogastric Junction Cancers, Version 2.2019,NCCN Clinical Practice Guidelines in Oncology. J Natl Compr Canc Netw (2019) 17 (7)855-83. doi: 10.6004/jnccn.2019.0033

3. Deng W, Lin SH. Advances in radiotherapy for esophageal cancer. Ann Transl Med (2018) 6(4):79. doi: 10.21037/atm.2017.11.28

4. Tonison JJ, Fischer SG, Viehrig M, Welz S, Boeke S, Zwirner K, et al. Radiation pneumonitis after intensity-modulated radiotherapy for esophageal cancer: institutional data and a systematic review. Sci Rep (2019) 9(1):2255. doi: 10.1038/s41598-018-38414-5

5. Zhang Y, Wang H, Huang X, Zhang Q, Ren R, Sun R, et al. Dosimetric comparison of TomoDirect, helical tomotherapy, VMAT, and ff-IMRT for upper thoracic esophageal carcinoma. Med Dosim (2019) 44(2):167-72. doi: 10.1016/j.meddos.2018.05.001 diagnosis and treatment of diseases and the prediction of complications.

\section{CONCLUSIONS}

CT radiomics has powerful data-processing and analysis abilities. In this context, we explored a method to predict RP based on the lung $\mathrm{CBCT}$ radiomics features for EC patients. More importantly, we used this method to successfully build and validate a novel nomogram with good predictive value, which can help clinicians identify high-risk RP patients early and guide personalized treatment and clinical decisions.

\section{DATA AVAILABILITY STATEMENT}

The raw data supporting the conclusions of this article will be made available by the authors, without undue reservation.

\section{AUTHOR CONTRIBUTIONS}

FD and NT are responsible for analyzing data and writing papers. JL designed experiments to guide the writing and revision of papers. WW directed the writing and revision of papers. YC, YZ, and ZL were responsible for radiomics diagnosis and radiomics data processing of patients. All authors contributed to the article and approved the submitted version.

\section{FUNDING}

Funding was obtained from the National Key Research Program of China (No. 2016YFC0904700), the National Natural Science Foundation of China (No. 81773287), and The Key Research Development Program of Shandong Province (No. 2016GSF201093).

6. Cho WK, Oh D, Kim HK, Ahn YC, Noh JM, Shim YM, et al. Dosimetric predictors for postoperative pulmonary complications in esophageal cancer following neoadjuvant chemoradiotherapy and surgery. Radiother Oncol (2019) 133:87-92. doi: 10.1016/j.radonc.2019.01.005

7. Rodrigues G, Lock M, D'Souza D, Yu E, Dyk JV. Prediction of radiation pneumonitis by dose-volume histogram parameters in lung cancer-A systematic review. Radiother Oncol (2004) 71(2):127-38. doi: 10.1016/j.radonc.2004.02.015

8. Senan S, Cardenal F, Vansteenkiste J, Stigt J, Akyol F, Neve WD. A randomized phase II study comparing induction or consolidation chemotherapy with cisplatindocetaxel,plus radical concurrent chemoradiotherapy with cisplatin-docetaxel,in patients with unresectable locally advanced non-small-cell lung cancer. Ann Oncol (2011) 22:553-8. doi: 10.1093/annonc/mdq388

9. Graham M V, Purdy JA, Emami B, Harms W, Bosch W, Lockett MA. Clinical dose-volume histogram analysis for pneumonitis after 3D treament for nonsmall cell lung cancer(NSCLC). Int J Radiat Oncol Biol Phys (1999) 45(2):3239. doi: 10.1016/s0360-3016(99)00183-2

10. Bashir U, Siddique MM, Mclean E, Goh V, Cook GJ. Imaging heterogeneity in lung cancer:techniques,applications, and challenges. Am J Roentgenol (2016) 207(3):534-43. doi: 10.2214/AJR.15.15864 
11. Yip SS, Aerts HJ. Applications and limitations of radiomics. Phys Med Biol (2016) 61(13):R150-66. doi: 10.1088/0031-9155/61/13/R150

12. Cunliffe A, Armato SG, Castillo R, Pham N, Guerrero T, Al-Hallaq HA. Lung texture in serial thoracic computed tomography scans:correlation of radiomics-based features with radiation therapy dose and radiation pneumonitis development. Int J Radiat Oncol Biol Phys (2015) 91(5):104856. doi: 10.1016/j.ijrobp.2014.11.030

13. Krafft SP, Rao A, Stingo F, Briere TM, Court LE, Liao Z, et al. The utility of quantitative CT radiomics features for improved prediction of radiation pneumonitis. Med Phys (2018) 45(11):5317-24. doi: 10.1002/mp.13150

14. Lambin P, Rios-Velazquez E, Leijenaar R, Carvalho S, van Stiphout RG, Granton P, et al. Radiomics: Extracting more information from medical images using advanced feature analysis. Eur J Cancer (2012) 48(4):441-6. doi: 10.1016/j.ejca.2011.11.036

15. Gillies RJ, Kinahan PE, Hricak H. Radiomics:images are more than pictures, they are data. Radiology (2016) 278(2):563-77. doi: 10.1148/radiol.2015151169

16. Parmar C, Rios Velazquez E, Leijenaar R, Jermoumi M, Carvalho S, Mak RH, et al. Robust radiomics feature quantification using semiautomatic volumetric segmentation. PloS One (2014) 9(7):e102107. doi: 10.1371/journal.pone.0102107

17. Doumou G, Siddiques M, Tsoumpas C, Goh V, Cook GJ. The precision of textural analysis in (18)F-FDG-PET scans of oesophageal cancer. Eur Radiol (2015) 25(9):2805-12. doi: 10.1007/s00330-015-3681-8

18. Coroller TP, Grossmann P, Hou Y, Velazquez ER, Leijenaar RTH, Hermann G, et al. CT-based radiomic signature predicts distant metastasis in lung adenocarcinoma. Radiother Oncol (2015) 114(3):345-50. doi: 10.1016/j.radonc.2015.02.015

19. Tibshirani R. Regression Shrinkage and Selection via the Lasso:a retrospective. J Roy Statist Soc Ser B (1996) 58(1):267-88. doi: 10.1111/j.1467-9868. 2011.00771.x

20. Gui J, Li H. Penalized Cox regression analysis in the high-dimensional and low-sample size settings, with applications to microarray gene expression data. Bioinformatics (2005) 21(13):3001-8. doi: 10.1093/bioinformatics/bti422

21. Hepp T, Schmid M, Gefeller O, Waldmann E, Mayr A. Approaches to regularized regression-a comparison between gradient boosting and the Lasso. Methods Inf Med (2016) 55(5):422-30. doi: 10.3414/ME16-01-0033

22. Ndhlovu ZM, Chibnik LB, Proudfoot J, Vine S, McMullen A, Cesa K, et al. High-dimensionai immunomonitonitoring models of HIV-1-specific CD8 Tcell responses accurately identify subjects achieving spontaneous viral control. Blood (2013) 121(5):801-11. doi: 10.1182/blood-2012-06-436295

23. Kumamaru KK, Saboo SS, Aghayev A, Cai P, Quesada CG, George E, et al. CT pulmonary angiography-based scoring system to predict the prognosis of acute pulmonary embolism. J Cardiovasc Comput Tomogr (2016) 10(6):473-9. doi: $10.1016 /$ j.jcct.2016.08.007

24. Vasquez MM, Hu C, Roe DJ, Chen Z, Halonen M, Guerra S. Least absolute shrinkage and selection operator type methods for the identification of serum biomarkers of overweight and obesity: simulation and application. BMC Med Res Methodol (2016) 16(1):154. doi: 10.1186/s12874-016-0254-8

25. Xu C-J, van der Schaaf A, van't Veld AA, Langendijk JA, Schilstra C. Statistical validation of normal tissue complication probability models. Int J Radiat Oncol Biol Phys (2012) 84(1):e123-9. doi: 10.1016/j.ijrobp.2012.02.022

26. Friedman J, Hastie T, Tibshirani R. Regularization Paths for Generalized Linear Models via Coordinate Descent. J Stat software (2010) 33(1):1-22. doi: 10.1016/j.jspi.2009.07.020

27. Aerts HJ, Velazquez ER, Leijenaar RT, Parmar C, Grossmann P, Carvalho S, et al. Decoding tumour phenotype by noninvasive imaging using aquantitative radiomics approach. Nat Commun (2014) 5:4006. doi: 10.1038/ncomms5006

28. Jenkins $\mathrm{P}$, Welsch A. Computed tomography appearance of early radiation injury to the lung: Correlation with clinical and dosimetric factors. Int J Radiat Oncol Biol Phys (2011) 81(1):97-103. doi: 10.1016/j.ijrobp.2010.05.017

29. Xu L, Jiang J, Li Y, Zhang L, Li Z, Xian J, et al. Genetic variants of SP-D confer susceptibility to radiation pneumonitis in lung cancer patients undergoing thoracic radiation therapy. Cancer Med (2019) 8(5):2599-611. doi: 10.1002/cam4.2088

30. Yin M, Liao Z, Liu Z, Wang L, O'Reilly M, Gomez D, et al. Genetic variants of the nonhomologous end joining gene LIG4 and severe radiation pneumonitis in nonsmall cell lung cancer patients treated with definitive radiotherapy. Cancer (2012) 118:528-35. doi: 10.1002/cncr.26214

31. Mackin D, Fave XB, Zhang L, Fried D, Yang J, Taylor B, et al. Measuring computed tomography scanner variability of radiomics features. Invest Radiol (2015) 50:(11):757-65. doi: 10.1097/RLI.0000000000000180
32. Daghir-Wojtkowiak E, Wiczling P, Bocian S, Kubik Ł, Kośliński P, Bocian S, et al. Least absolute shrinkage and selection operator and dimensionality reduction techniques in quantitative structure retention relationship modeling of retention in hydrophilic interaction liquid chromatography. J Chromatogr A (2015) 1403:54-62. doi: 10.1016/j.chroma.2015.05.025

33. Tucker S L, Liao Z, Dinh J, Bian SX, Mohan R, Martel MK, et al. Is there an impact of heart exposure on the incidence of radiation pneumonitis? Analysis of data from a large clinical cohort. Acta Oncol (2014) 53(5):590-6. doi: 10.3109/0284186X.2013.831185

34. Zhao J, Yorke ED, Li L, Kavanagh BD, Li XA, Das S, et al. Simpe factors associated with radiatio-reduced lung toxicity after stereotactic body radiation therapy of the thorax:a pooled analysis of 88 studies. Int J Radiat Oncol Biol Phys (2016) 95(5):1357-66. doi: 10.1016/j.ijrobp.2016.03.024

35. Chargari C, Riet F, Mazevet M, Morel E, Lepechoux C, Deutsch E. Complications of thoracic radiotherapy. Presse Med (2013) 42 (9 pt 2):e34251. doi: 10.1016/j.lpm.2013.06.012

36. Tucker S L, Liu HH, Liao Z, Wei X, Wang S, Jin H, et al. Analysis of radiation pneumonitis risk using a generalized lyman model. Int J Radiat Oncol Biol Phys (2008) 72(2):568-74. doi: 10.1016/j.ijrobp.2008.04.053

37. Jin H, Tucker SL, Liu HH, Wei X, Yom SS, Wang S, et al. Dose-volume thresholds and smoking status for the risk of treatment-related pneumonitis in inoperable non-small cell lung cancer treated with definitive radiotherapy. Radiother Oncol (2009) 919(3):427-32. doi: 10.1016/j.radonc.2008.09.009

38. Takeda A, Kunieda E, Ohashi T, Aoki Y, Oku Y, Enomoto T, et al. Severe COPD is correlated with mild radiation pneumonitis following stereotactic body radiotherapy. Chest (2012) 141(4):858-66. doi: 10.1378/chest.11-1193

39. Kimura T, Togami T, Takashima H, Nishiyama Y, Ohkawa M, Nagata Y. Radiation pneumonitis in patients with lung and mediastinal tumours:a retrospective study of risk factors focused on pulmonary emphysema. $\mathrm{Br} \mathrm{J}$ Radiol (2012) 85(1010):135-41. doi: 10.1259/bjr/32629867

40. Permuth JB, Choi J, Balarunathan Y, Kim J, Chen DT, Chen L, et al. Combining radiomic features with a miRNA classififier may improve prediction of malignant pathology for pancreatic intraductal papillary mucinous neoplasms. Oncotarget (2016) 7:85785-97. doi: 10.18632/ oncotarget.11768

41. Wang L, Liang S, Li C, Sun X, Pang L, Meng X, et al. A novel nomogram and risk classification system predicting radiation pneumonitis in patients with esophageal cancer receiving radiotherapy. Int J Radiat Oncol Biol Phys (2019) 105(5):1074-85. doi: 10.1016/j.ijrobp.2019.08.024

42. Dreiseitl S, Ohno-Machado L. Logistic regression and artificial neural network classifications models: A methodology review. J BioMed Inform (2002) 35(56):352-9. doi: 10.1016/s1532-0464(03)00034-0

43. Ayer T, Chhatwal J, Alagoz O, Jr CEK, Woods RW, Burnside ES. Informatics in radiology:Comparison of logistic regression and artificial neural network models in breast cancer risk estimation. Radiographics (2010) 30(1):13-22. doi: 10.1148/rg.301095057

44. Coy S L, Cheema AK, Tyburski JB, Laiakis EC, Collins SP, Fornace A Jr. Radiation metabolomics and its potential in biodosimetry. Int J Radiat Biol (2011) 87(8):802-23. doi: 10.3109/09553002.2011.556177

45. Hoover DA, Reid RH, Wong E, Stitt L, Sabondjian E, Rodrigues GB, et al. SPECT-based functional lung imaging for the prediction of radiation pneumonitis: A clinical and dosimetric correlation. J Med Imaging Radiat Oncol (2014) 58(2):214-22. doi: 10.1111/1754-9485.12145

46. Castillo R, Pham N, Ansari S, Meshkov D, Castillo S, Li M, et al. Preradiotherapy FDG PET predicts radiation pneumonitis in lung cancer. Radiat Oncol (2014) 9:74. doi: 10.1186/1748-717X-9-74

Conflict of Interest: The authors declare that the research was conducted in the absence of any commercial or financial relationships that could be construed as a potential conflict of interest.

Copyright (c) $2020 \mathrm{Du}$, Tang, Cui, Wang, Zhang, Li and Li. This is an open-access article distributed under the terms of the Creative Commons Attribution License (CC BY). The use, distribution or reproduction in other forums is permitted, provided the original author(s) and the copyright owner(s) are credited and that the original publication in this journal is cited, in accordance with accepted academic practice. No use, distribution or reproduction is permitted which does not comply with these terms. 